\title{
Genome-Wide DNA Methylation Study Identifies Significant Epigenomic Changes in Osteoarthritic Cartilage
}

\author{
Matlock A. Jeffries, ${ }^{1}$ Madison Donica, ${ }^{2}$ Lyle W. Baker, ${ }^{3}$ Michael E. Stevenson, ${ }^{4}$ \\ Anand C. Annan, ${ }^{4}$ Mary Beth Humphrey, ${ }^{5}$ Judith A. James, ${ }^{1}$ and Amr H. Sawalha ${ }^{6}$
}

\begin{abstract}
Objective. To perform a genome-wide DNA methylation study to identify DNA methylation changes in osteoarthritic (OA) cartilage tissue.

Methods. The contribution of differentially methylated genes to $\mathrm{OA}$ pathogenesis was assessed by bioinformatic analysis, gene expression analysis, and histopathologic severity correlation. Genome-wide DNA methylation profiling of $>485,000$ methylation sites was performed on eroded and intact cartilage from within the same joint of 24 patients undergoing hip arthroplasty for OA. Genes with differentially methylated CpG sites were analyzed to identify overrepresented gene ontologies, pathways, and upstream regulators. The messenger RNA expression of a subset of differentially methylated genes was analyzed by reverse transcription-polymerase chain reaction. Histopathology was graded by modified Mankin score and correlated with DNA methylation.
\end{abstract}

Supported by the Rheumatology Research Foundation Ephraim P. Engleman Endowed Resident Research Preceptorship and the McBride Foundation Arthritis Research Grant (both to Dr. Jeffries).

${ }^{1}$ Matlock A. Jeffries, MD, Judith A. James, MD, PhD: University of Oklahoma Health Sciences Center and Oklahoma Medical Research Foundation, Oklahoma City; ${ }^{2}$ Madison Donica, BS: Oklahoma Medical Research Foundation, Oklahoma City; ${ }^{3}$ Lyle W. Baker, BA: University of Oklahoma, Oklahoma City; ${ }^{4}$ Michael E. Stevenson, MD, MPH, Anand C. Annan, MD: University of Oklahoma Health Sciences Center, Oklahoma City; ${ }^{5}$ Mary Beth Humphrey, MD, PhD: University of Oklahoma Health Sciences Center, Oklahoma Medical Research Foundation, and Department of Veterans Affairs Medical Center, Oklahoma City, Oklahoma; ${ }^{6} \mathrm{Amr} \mathrm{H}$. Sawalha, MD: Oklahoma Medical Research Foundation, Oklahoma City, and University of Michigan, Ann Arbor.

Address correspondence to Matlock A. Jeffries, MD, Oklahoma Medical Research Foundation, 825 NE 13th Street, MC400, Oklahoma City, OK 73104 (e-mail: matlock-jeffries@ouhsc.edu); or to Amr H. Sawalha, MD, University of Michigan, Division of Rheumatology, Department of Internal Medicine, 5520 MSRB-1, SPC 5680, 1150 West Medical Center Drive, Ann Arbor, MI 48109 (e-mail: asawalha@umich.edu).

Submitted for publication January 9, 2014; accepted in revised form June 24, 2014.
Results. We identified 550 differentially methylated sites in OA. Most (69\%) were hypomethylated and enriched among gene enhancers. We found differential methylation in genes with prior links to OA, including RUNX1，RUNX2，DLX5，FURIN，HTRA1，FGFR2, NFATC1, SNCAIP, and COL11A2. Among these, RUNX1, HTRA1, FGFR2, and COL11A2 were also differentially expressed. Furthermore, we found differential methylation in approximately one-third of known OA susceptibility genes. Among differentially methylated genes, upstream regulator analysis showed enrichment of TGFB1 $\left(P=4.40 \times 10^{-5}\right)$ and several microRNAs including miR-128 $\left(P=4.48 \times 10^{-13}\right)$, miR-27a $(P=$ $\left.4.15 \times 10^{-12}\right)$, and miR-9 $\left(P=9.20 \times 10^{-10}\right)$. Finally, we identified strong correlations between 20 CpG sites and the histologic Mankin score in OA.

Conclusion. Our data implicate epigenetic dysregulation of a host of genes and pathways in OA, including a number of $\mathrm{OA}$ susceptibility genes. Furthermore, we identified correlations between CpG methylation and histologic severity in OA.

Osteoarthritis (OA) is a chronic, debilitating musculoskeletal disease characterized by progressive loss of function of both load-bearing and non-loadbearing joints, leading to significant pain and functional limitation. Although a hallmark of OA is loss of hyaline cartilage leading to joint space narrowing, other articular tissues and processes have been implicated, including synovial inflammation and subchondral bone remodeling. An unrecognized epidemic, OA is the leading cause of chronic disability in the US, affecting $40 \%$ of US adults age $>70$ years (1). Indeed, the risk of disability resulting from knee or hip $\mathrm{OA}$ is equivalent to that resulting from cardiovascular disease (2). Recent estimates place the annual cost of OA at $\$ 128$ billion in the US, representing nearly $1 \%$ of gross domestic product (3). Alarmingly, the prevalence of severe OA requiring 
joint replacement is increasing at a rate that exceeds expected increases due to obesity and aging (4).

Unlike many other rheumatic diseases, relatively little is known about the pathophysiology of OA. Consequently, treatment options remain diminutive, consisting principally of analgesic agents and viscosupplementation. Remarkably, although the incidence of OA is 400-fold higher than that of rheumatoid arthritis (RA) (5), there have been no disease-modifying agents approved for the treatment of OA. More must be done to understand the mechanisms involved in the development and progression of OA to move the field toward novel therapeutic strategies.

The pathogenesis of OA involves the interplay of 3 major factors: genetic predisposition, aging, and the environment (6). Genome-wide association studies have identified surprisingly few candidate genetic susceptibility loci, the majority of which are within either structural genes or cellular signaling pathways (7-9). Several studies have recently implicated epigenetic dysregulation as a contributor to OA pathology. Epigenetics, defined as heritable changes in gene expression that occur in the absence of mutations of underlying genomic DNA, is a common mechanism whereby organisms alter gene expression in response to both external and internal environmental cues. Epigenetic regulation occurs by a few common mechanisms, including genomic DNA methylation, alterations of histone side chains resulting in chromatin conformational change, and noncoding RNA feedback. Epigenetic dysregulation leading to inappropriate gene expression or silencing has been shown to play an important role in several rheumatic diseases, such as systemic lupus erythematosus (10-12) and RA (13-15).

The most widely studied epigenetic control mechanism is DNA methylation. The addition of methyl groups to the $5^{\prime}$ position of cytosine in fully differentiated tissues most commonly occurs in $\mathrm{CpG}$ dinucleotides, mediated by a variety of DNA methyltransferases. Methylation of certain regulatory regions is of particular transcriptional relevance, most commonly within the $5^{\prime}$-untranslated region (5'-UTR) or 5'-promoter region upstream of a target gene or located near or within $\mathrm{CpG}$-enriched regions known as $\mathrm{CpG}$ islands. Methylated cytosines within these areas tend to be transcriptionally repressive, whereas unmethylated cytosines are permissive for gene expression. To date, few studies have been undertaken to examine the role of epigenetics in OA, but they have proven quite fruitful. Candidate gene methylation analyses in OA cartilage have identified significant alterations in DNA methylation signa- tures of the matrix metalloproteinases (MMPs) MMP3, MMP6, MMP9, MMP13 (16), and ADAMTS4 (17), and, curiously, the obesity- and inflammation-linked leptin gene (18). A recent report described a genome-wide DNA methylation analysis that examined the status of $\sim 27,000 \mathrm{CpG}$ sites in OA knee cartilage compared to normal control cartilage and identified a number of differentially methylated $\mathrm{CpG}$ sites as well as a cluster of cases that appeared enriched in inflammatory genes (19).

Our present work sought to expand this knowledge of OA epigenetics in order to address 2 major questions unanswered by previous studies. The first was whether regional differences in methylation exist between eroded and intact cartilage within the same joint, which may be linked to disease progression. We hypothesized that many genes and pathways linked to OA would have significant changes in their epigenetic state as the disease progresses. Our second question was whether $\mathrm{CpG}$ methylation varies linearly with OA disease activity; we hypothesized that several differentially methylated $\mathrm{CpG}$ sites would be highly correlated with histologic score. To address these questions, we performed the largest survey, pathway, and ontological analysis to date of the methylation status of $>485,000$ $\mathrm{CpG}$ sites throughout the entire genome of paired samples of eroded and intact cartilage obtained from hip joints of 24 OA patients. Additionally, we performed histopathologic modified Mankin scoring (20) and methylation correlation analyses on 12 samples, as well as limited gene expression profiling of selected differentially methylated genes in a subset of 12 samples.

\section{MATERIALS AND METHODS}

Samples and nucleic acid isolation. Twenty-four femoral heads were obtained from patients undergoing hip arthroplasty for end-stage primary OA at the McBride Orthopedic Hospital in Oklahoma City. Demographic information about these patients is presented in Supplementary Table 1, available on the Arthritis \& Rheumatology web site at http://online library.wiley.com/doi/10.1002/art.38762/abstract. The Institutional Review Boards in all involved institutions approved this study. Full-thickness cartilage samples representing grossly eroded and grossly intact cartilage were obtained from areas of each femoral head, divided into portions for DNA and RNA extraction, and flash-frozen in liquid nitrogen. Additional portions of tissue were collected from 6 femoral heads and placed in $4 \%$ paraformaldehyde (Sigma-Aldrich) for subsequent histologic analysis. Samples were then ground using either a mortar and pestle or a cryogenic grinder mill (Spex CertiPrep). DNA was isolated from each sample using a DNeasy kit (Qiagen), and 500 ng was subsequently treated with sodium bisulfite using an EZ-DNA MethylationLightning kit (Zymo Research). 
DNA methylation studies. Genome-wide DNA methylation was assessed using the HumanMethylation 450 BeadChip microarray (Illumina), which analyzes the methylation status of $>485,000$ methylation sites throughout the genome, covering $99 \%$ of RefSeq genes at an average of $17 \mathrm{CpG}$ sites per gene across the $5^{\prime}$-UTR, gene promoter regions, first exon, gene body, and $3^{\prime}$-UTR, and covering $96 \%$ of University of California, Santa Cruz-defined $\mathrm{CpG}$ islands and their flanking regions. Following bisulfite treatment, DNA from each sample was loaded onto chips and processed by the Oklahoma Medical Research Foundation Genotyping Core Facility. This array includes a variety of both sample-independent and sample-dependent controls, which were evaluated in each chip.

Histologic examination. Following fixation, samples were embedded in paraffin and slides were cut for both hematoxylin and eosin and Safranin O staining. Two pathologists (MES, ACA) scored each case using the modified Mankin scale, and a mean score was determined (see Supplementary Table 2, available on the Arthritis \& Rheumatology web site at http://onlinelibrary.wiley.com/doi/10.1002/art.38762/abstract).

Statistical analysis. Chips were imaged and data were extracted using the GenomeStudio methylation module, version 2011.1 (Illumina). The percent methylated cytosines at each $\mathrm{CpG}$ site $(\beta)$ was calculated as the ratio of methylated probe signal to total locus signal intensity and defined within a range of 0 to 1 by GenomeStudio; average $\beta$ values were then compared. Differentially methylated $\mathrm{CpG}$ sites were defined as those having a differential methylation score $\geq|21|$, corresponding to a $P$ value of less than 0.01 after adjusting for multiple testing using GenomeStudio's built-in false discovery rate function, which employs a Benjamini and Hochberg procedure with $\alpha=0.05$. A second requirement was a mean methylation difference $(\Delta \beta) \geq 0.15(15 \%)$ between the 2 groups. $\mathrm{CpG}$ sites with a known single-nucleotide polymorphism located within or $10 \mathrm{bp}$ from the $3^{\prime}$-end of the probe were excluded. The locations of particular $\mathrm{CpG}$ sites within regulatory regions were defined by GenomeStudio, including $\mathrm{CpG}$ islands as well as North $\left(5^{\prime}\right)$ and South $\left(3^{\prime}\right)$ Shores (N-Shores and S-Shores) and North $\left(5^{\prime}\right)$ and South $\left(3^{\prime}\right)$ Shelves (N-Shelves and S-Shelves), which represent $\sim 2 \mathrm{~kb}$ surrounding $\mathrm{CpG}$ islands and shores, respectively. Location statistics were calculated using the Yates' correction of a chi-square distribution $P$ value of a $2 \times 2$ contingency table.

Gene ontology analyses. Functional properties, networks, pathways, and upstream regulators enriched in differentially methylated genes were assessed using the Ingenuity Pathways Analysis (IPA) system (Ingenuity Systems) using the Ingenuity Knowledge Base reference set. Direct and indirect relationships were calculated, and experimentally observed relationships were included; $P$ values less than or equal to 0.05 were considered significant. In addition to the standard upstream regulator analysis, microRNAs (miRNAs) overrepresented among differentially methylated genes were identified by IPA using experimentally demonstrated and predicted miRNA-messenger RNA (mRNA) interactions or binding sites from TarBase, miRecords, and TargetScan databases, as well as peer-reviewed miRNA research articles as curated by Ingenuity Systems staff.

Histologic correlation. In the 12 samples with histopathologic scores, $\mathrm{CpG}$ methylation at each site was compared to the modified Mankin score. The coefficient of determina- tion $\left(\mathrm{R}^{2}\right)$ was calculated using a linear regression model. Correlations were deemed significant if $\mathrm{R}^{2}$ was $>0.85$ and the range of methylation values across samples for the $\mathrm{CpG}$ was at least $15 \%\left(\Delta \beta_{\text {samples }} \geq 0.15\right)$.

Reverse transcription-polymerase chain reaction (RTPCR). The expression profiles of several of the most differentially hypomethylated and hypermethylated genes identified in our assay were evaluated by real-time RT-PCR. RNA was isolated using an Ambion RNAqueous kit (Life Technologies) in accordance with the manufacturer's instructions. Real-time RT-PCR was performed with a RotorGene 3000 (Qiagen) using an iScript one-step RT-PCR kit (Bio-Rad) with the following cycling conditions: 30 minutes at $50^{\circ} \mathrm{C}, 15$ minutes at $95^{\circ} \mathrm{C}, 70$ cycles of 15 seconds at $94^{\circ} \mathrm{C}$ and 20 seconds at $56^{\circ} \mathrm{C}$, and 30 seconds at $72^{\circ} \mathrm{C}$. Primer sequences are listed in Supplementary Table 3, available on the Arthritis \& Rheumatology web site at http://onlinelibrary.wiley.com/doi/10.1002/ art.38762/abstract. Relative mRNA concentrations were calculated by RotorGene6 software, using the $\Delta \Delta \mathrm{C}_{\mathrm{t}}$ method with $G A P D H$ as a housekeeping gene. GAPDH $\mathrm{C}_{\mathrm{t}}$ values were similar among eroded and intact cartilage samples.

Array confirmation. DNA methylation values of selected hypomethylated, intermediate-methylated, and hypermethylated CpGs within $H O X A 3, H O X A 5$, and $H O X A 9$ were determined by traditional bisulfite Sanger sequencing and subsequent analysis using the ESME software package (Epigenomics) (21), a method we have used extensively in the past (10). We found a high level of correlation between methylation values reported by the Illumina array and those found by this traditional sequencing method $\left(\mathrm{R}^{2}=0.84 ; \mathrm{n}=193\right)$ (see Supplementary Figure 1, available on the Arthritis \& Rheumatology web site at http://onlinelibrary.wiley.com/doi/10.1002/ art.38762/abstract).

Differential methylation confirmation. Finally, to confirm differential methylation of $3 \mathrm{CpG}$ sites among the most highly differentially hypomethylated genes identified by the Illumina array (cg19348484: FURIN; $\operatorname{cg} 04915566$ and cg01519261: RUNX1), custom, commercial pyrosequencing analysis was performed (EpigenDx) on a subset of 12 eroded and 12 intact cartilage samples.

\section{RESULTS}

Significant differential CpG methylation between eroded and intact $\mathrm{OA}$ cartilage. We identified a total of 550 differentially methylated CpG sites in grossly eroded cartilage samples compared with intact samples. Of these, 378 (69\%) were hypomethylated and 172 (31\%) were hypermethylated (Table 1 and Figure 1), corresponding to 390 distinct, known genes and nearby genomic regions. Differentially methylated sites were concentrated in enhancers, 50\% observed among hypomethylated sites $(P<0.0001)$ and $37 \%$ observed among hypermethylated CpGs $(P=0.002)$, as compared to $21 \%$ expected for both for the assay. Enhancers are segments of regulatory genomic sequence that may exert effects at remote locations to control both spatial and 
Table 1. Top CpG sites differentially hypomethylated and hypermethylated in eroded compared to intact cartilage*

\begin{tabular}{|c|c|c|c|c|c|c|c|c|c|}
\hline $\begin{array}{l}\text { CpG site methylation } \\
\text { status, Illumina } \mathrm{CpG} \\
\text { ID no. }\end{array}$ & $\begin{array}{l}\text { Associated } \\
\text { gene } \\
\text { symbol }\end{array}$ & $\begin{array}{c}\text { Mean } \beta \text {, } \\
\text { eroded }\end{array}$ & $\begin{array}{l}\text { Mean } \beta \text {, } \\
\text { intact }\end{array}$ & $\Delta \beta$ & $\begin{array}{c}\text { Differential score, } \\
\text { FDR corrected }\end{array}$ & $\begin{array}{l}P, \text { FDR } \\
\text { corrected }\end{array}$ & $\begin{array}{l}\text { UCSC } \\
\text { location } \\
\text { group }\end{array}$ & $\begin{array}{l}\text { UCSC } \\
\text { island } \\
\text { group }\end{array}$ & $\begin{array}{l}\text { Located in } \\
\text { enhancer }\end{array}$ \\
\hline \multicolumn{10}{|l|}{ Hypomethylated } \\
\hline cg19348484 & FURIN & 0.39 & 0.68 & -0.30 & -347.21 & $2.00 \times 10^{-38}$ & 5'-UTR & N-Shore & Yes \\
\hline $\operatorname{cg} 04915566$ & $R U N X 1$ & 0.24 & 0.52 & -0.27 & -347.21 & $2.00 \times 10^{-38}$ & 5'-UTR & - & - \\
\hline $\operatorname{cg} 03278514$ & NA & 0.26 & 0.53 & -0.27 & -347.21 & $2.00 \times 10^{-38}$ & - & - & Yes \\
\hline cg25388800 & NA & 0.21 & 0.47 & -0.26 & -347.21 & $2.00 \times 10^{-38}$ & - & - & Yes \\
\hline $\operatorname{cg} 18873386$ & $D L X 5$ & 0.47 & 0.73 & -0.26 & -347.21 & $2.00 \times 10^{-38}$ & Gene body & N-Shore & - \\
\hline $\operatorname{cg} 01519261$ & $R U N X 1$ & 0.32 & 0.57 & -0.26 & -347.21 & $2.00 \times 10^{-38}$ & $5^{\prime}$-UTR & - & - \\
\hline $\operatorname{cg} 25928986$ & NA & 0.37 & 0.62 & -0.25 & -347.21 & $2.00 \times 10^{-38}$ & - & S-Shore & - \\
\hline cg16017420 & NA & 0.41 & 0.66 & -0.25 & -347.21 & $2.00 \times 10^{-38}$ & - & S-Shelf & - \\
\hline cg13784312 & $R A P G E F 1$ & 0.37 & 0.62 & -0.25 & -347.21 & $2.00 \times 10^{-38}$ & Gene body & - & - \\
\hline $\operatorname{cg} 12534147$ & NA & 0.27 & 0.52 & -0.24 & -347.21 & $2.00 \times 10^{-38}$ & - & - & Yes \\
\hline $\operatorname{cg} 12686441$ & NA & 0.29 & 0.53 & -0.23 & -347.21 & $2.00 \times 10^{-38}$ & - & - & - \\
\hline cg08369079 & PRDM1 & 0.43 & 0.66 & -0.23 & -347.21 & $2.00 \times 10^{-38}$ & Gene body & - & - \\
\hline cg11756029 & TRAFD1 & 0.30 & 0.53 & -0.23 & -347.21 & $2.00 \times 10^{-38}$ & Gene body & - & - \\
\hline $\operatorname{cg} 10193711$ & NA & 0.31 & 0.54 & -0.23 & -347.21 & $2.00 \times 10^{-38}$ & - & - & - \\
\hline cg27016494 & $D L X 5$ & 0.53 & 0.76 & -0.23 & -347.21 & $2.00 \times 10^{-38}$ & Gene body & N-Shore & - \\
\hline $\operatorname{cg} 16152753$ & NA & 0.36 & 0.58 & -0.22 & -347.21 & $2.00 \times 10^{-38}$ & - & S-Shelf & - \\
\hline $\operatorname{cg} 09966895$ & ODZ4 & 0.21 & 0.44 & -0.22 & -347.21 & $2.00 \times 10^{-38}$ & Gene body & - & Yes \\
\hline cg15840891 & NA & 0.23 & 0.45 & -0.22 & -347.21 & $2.00 \times 10^{-38}$ & - & - & Yes \\
\hline $\operatorname{cg} 05101437$ & CDK6 & 0.33 & 0.55 & -0.22 & -347.21 & $2.00 \times 10^{-38}$ & Gene body & - & Yes \\
\hline $\operatorname{cg} 01510278$ & NA & 0.28 & 0.49 & -0.22 & -347.21 & $2.00 \times 10^{-38}$ & - & - & Yes \\
\hline cg05973398 & $R U N X 1$ & 0.26 & 0.47 & -0.22 & -347.21 & $2.00 \times 10^{-38}$ & Gene body & - & Yes \\
\hline \multicolumn{10}{|l|}{ Hypermethylated } \\
\hline $\operatorname{cg} 04152616$ & $\mathrm{NA}$ & 0.58 & 0.38 & 0.19 & 349.79 & $1.05 \times 10^{-38}$ & - & & \\
\hline $\operatorname{cg} 27317046$ & $L T B P 2$ & 0.48 & 0.28 & 0.19 & 349.79 & $1.05 \times 10^{-38}$ & Gene body & - & Yes \\
\hline cg12226006 & FGFR2 & 0.65 & 0.46 & 0.19 & 349.79 & $1.05 \times 10^{-38}$ & Gene body & - & Yes \\
\hline cg22251148 & NA & 0.49 & 0.30 & 0.19 & 349.79 & $1.05 \times 10^{-38}$ & - & N-Shore & - \\
\hline $\operatorname{cg} 10707788$ & $G M D S$ & 0.58 & 0.38 & 0.20 & 349.79 & $1.05 \times 10^{-38}$ & Gene body & - & - \\
\hline cg00281273 & LPCAT1 & 0.45 & 0.26 & 0.20 & 349.79 & $1.05 \times 10^{-38}$ & Gene body & - & - \\
\hline $\operatorname{cg} 14856220$ & FGFR2 & 0.64 & 0.44 & 0.20 & 349.79 & $1.05 \times 10^{-38}$ & TSS 200 & N-Shelf & - \\
\hline $\operatorname{cg} 12528056$ & GPR44 & 0.51 & 0.31 & 0.20 & 349.79 & $1.05 \times 10^{-38}$ & 3'-UTR & Island & - \\
\hline cg24812143 & KIAA1274 & 0.52 & 0.32 & 0.20 & 349.79 & $1.05 \times 10^{-38}$ & Gene body & - & Yes \\
\hline cg14196395 & $D Y S F$ & 0.60 & 0.40 & 0.20 & 349.79 & $1.05 \times 10^{-38}$ & Gene body & - & - \\
\hline $\operatorname{cg} 05522011$ & PRDM8 & 0.65 & 0.45 & 0.20 & 349.79 & $1.05 \times 10^{-38}$ & Gene body & N-Shore & - \\
\hline cg23938483 & C14orf43 & 0.67 & 0.47 & 0.20 & 349.79 & $1.05 \times 10^{-38}$ & $3^{\prime}$-UTR & Island & - \\
\hline $\operatorname{cg} 10628201$ & $U B E 2 L 3$ & 0.49 & 0.28 & 0.20 & 349.79 & $1.05 \times 10^{-38}$ & Gene body & - & Yes \\
\hline cg20277356 & $F G F R 2$ & 0.72 & 0.51 & 0.22 & 349.79 & $1.05 \times 10^{-38}$ & TSS 200 & N-Shelf & - \\
\hline $\operatorname{cg} 00730832$ & $F G F R 2$ & 0.65 & 0.43 & 0.22 & 349.79 & $1.05 \times 10^{-38}$ & TSS 1,500 & N-Shelf & - \\
\hline $\operatorname{cg} 10314760$ & FGFR2 & 0.67 & 0.45 & 0.22 & 349.79 & $1.05 \times 10^{-38}$ & TSS 200 & N-Shelf & - \\
\hline cg14834653 & FGFR2 & 0.69 & 0.46 & 0.23 & 349.79 & $1.05 \times 10^{-38}$ & TSS 200 & N-Shelf & - \\
\hline cg17239876 & KIAA1274 & 0.51 & 0.28 & 0.23 & 349.79 & $1.05 \times 10^{-38}$ & Gene body & - & Yes \\
\hline $\operatorname{cg} 13175830$ & FGFR2 & 0.59 & 0.36 & 0.23 & 349.79 & $1.05 \times 10^{-38}$ & TSS 200 & N-Shelf & - \\
\hline cg24974704 & ZCЗH3 & 0.53 & 0.28 & 0.24 & 349.79 & $1.05 \times 10^{-38}$ & Gene body & - & - \\
\hline cg21036194 & SNCAIP & 0.86 & 0.61 & 0.26 & 349.79 & $1.05 \times 10^{-38}$ & Gene body & - & - \\
\hline
\end{tabular}

$* \Delta \beta=$ absolute difference in methylation value between sample groups $\left(\beta_{\text {eroded }}-\beta_{\text {intact }}\right)$; FDR $=$ false discovery rate; UCSC $=$ University of California, Santa Cruz; 5'-UTR $=5^{\prime}$-untranslated region; $N=$ North; NA $=$ not applicable; $S=$ South; TSS $200=$ within 200 bp of transcription start site.

temporal gene expression. Additional enrichment was noted in the $\mathrm{N}$-Shore regions of $\mathrm{CpG}$ islands among hypomethylated CpGs (9\% observed compared to 5\% expected; $P=0.03$ ) and within gene bodies among hypermethylated CpGs (68\% observed compared to $36 \%$ expected; $P<0.0001$ ) (see Supplementary Table 4, available on the Arthritis \& Rheumatology web site at http://onlinelibrary.wiley.com/doi/10.1002/art.38762/ abstract).
Differential methylation among several genes implicated in OA pathology. The most hypomethylated CpG site in our analysis was in the 5'-UTR of FURIN, encoding a proprotein convertase. Previous studies have found FURIN to be significantly overexpressed in OA cartilage (22), which is consistent with our methylation findings; however, we did not find evidence of overexpression in our samples (mean \pm SEM $2.4 \pm 0.8$ relative units for eroded cartilage versus $1.5 \pm 0.3$ relative units 


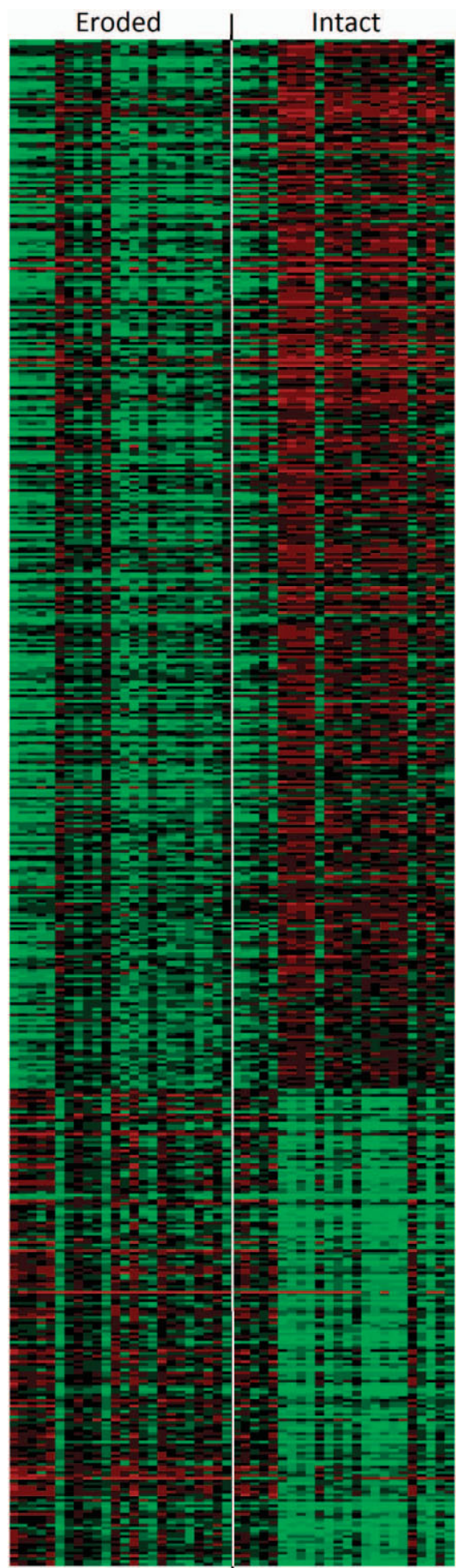

Figure 1. Heatmap of 550 differentially methylated $\mathrm{CpG}$ sites among eroded and intact cartilage samples from femoral heads of 24 patients with osteoarthritis. Rows represent differentially methylated $\mathrm{CpG}$ sites. Columns represent samples. Red indicates hypermethylated. Green indicates hypomethylated. for intact cartilage; $P=0.29$ ) (Figure 2D). Significant hypomethylation of this $\mathrm{CpG}$ site was independently confirmed by pyrosequencing analysis $(\operatorname{cg} 19348484: \Delta \beta=$ $0.35, P=1.7 \times 10^{-4}$ ) (see Supplementary Figure 2, available on the Arthritis \& Rheumatology web site at http:// onlinelibrary.wiley.com/doi/10.1002/art.38762/abstract).

The second and sixth most hypomethylated CpG sites were within the 5 '-UTR of RUNX1, previously identified as differentially methylated in OA. We identified 6 additional hypomethylated sites associated with this gene. Counterintuitively, we found an $\sim 50 \%$ reduction of expression in eroded cartilage $(1.2 \pm 0.2$ relative units versus $2.6 \pm 0.3$ relative units; $P=0.002$ ) (Figure $2 \mathrm{G})$. Significant hypomethylation of 2 RUNX1 CpG

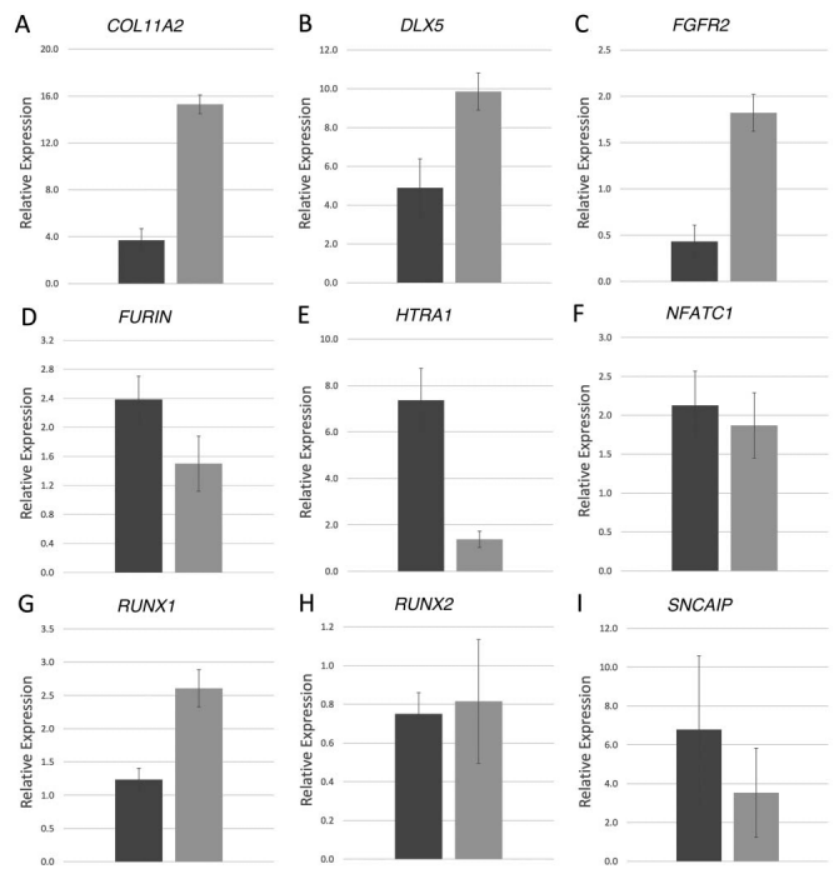

Figure 2. Relative mRNA expression of selected hypomethylated and hypermethylated genes associated with differentially methylated $\mathrm{CpG}$ sites, assessed by reverse transcription-polymerase chain reaction. Values are mean \pm SEM relative units for eroded cartilage (solid bars) versus relative units for intact cartilage (shaded bars), respectively. A, Hypermethylated COL11A2: $3.7 \pm 1$ versus $15 \pm 0.8(P=0.0002)$. B, Hypomethylated DLX5: $4.9 \pm 1$ versus $9.9 \pm 1(P=0.07)$. C, Hypermethylated FGFR2: $0.43 \pm 0.2$ versus $1.8 \pm 0.2(P=0.01)$. D, Hypomethylated FURIN: $2.4 \pm 0.8$ versus $1.5 \pm 0.3(P=0.29)$. E, Hypomethylated HTRA1: $7.4 \pm 1.4$ versus $1.4 \pm 0.4(P=0.002)$. F, Hypermethylated NFATC1: $2.13 \pm 0.4$ versus $1.87 \pm 0.4(P=$ 0.76). G, Hypomethylated RUNX1: $1.2 \pm 0.2$ versus $2.6 \pm 0.3$ $(P=0.002)$. H, Hypomethylated RUNX2: $0.75 \pm 0.1$ versus $0.82 \pm$ $0.3(P=0.9)$. I, Hypermethylated SNCAIP: $6.8 \pm 4$ versus $3.5 \pm 2$ $(P=0.6)$. 
sites was independently confirmed by pyrosequencing analysis $\left(\operatorname{cg} 04915566: \Delta \beta=0.28, P=5.1 \times 10^{-5}\right.$; $\operatorname{cg} 01519261: \Delta \beta=0.22, P=7.66 \times 10^{-5}$ ) (see Supplementary Figure 2, available on the Arthritis \& Rheumatology web site at http://onlinelibrary.wiley.com/doi/ 10.1002/art.38762/abstract). Our analysis also identified 2 hypomethylated CpG sites associated with $R U N X 2,1$ within a CpG island N-Shelf and 1 in the $5^{\prime}$-UTR of the gene. RUNX2 is highly associated with $\mathrm{OA}$ and is overexpressed in OA chondrocytes; however, we did not find a significant difference in expression of $R U N X 2$ among our samples $(0.75 \pm 0.1$ relative units for eroded cartilage versus $0.82 \pm 0.3$ relative units for intact cartilage; $P=0.9$ ) (Figure $2 \mathrm{H}$ ).

We identified 8 significantly hypomethylated CpG sites in both the body and within 1,500 bp of the transcription start site (TSS) of $D L X 5$, which plays an important role in chondrogenesis and induces the expression of Runx2 (23). Our studies indicate that DLX5 is epigenetically poised for expression; however, we did not find a significant difference in expression among samples $(4.9 \pm 1$ relative units for eroded cartilage versus $9.9 \pm 1$ relative units for intact cartilage; $P=$ 0.07) (Figure 2B). We found hypomethylation of $1 \mathrm{CpG}$ site within 1,500 bp of the TSS of HTRA1, a member of the high-temperature requirement family of serine proteases which is elevated in synovial fluid and cartilage of OA patients, and we found overexpression of HTRA1 (7.4 \pm 1.4 relative units for eroded cartilage versus $1.4 \pm$ 0.4 relative units for intact cartilage; $P=0.002$ ) (Figure $2 \mathrm{E}$ ), strongly suggesting epigenetic activation. Next, we found 2 significantly hypermethylated $\mathrm{CpGs}$ within an island and S-Shore associated with NFATC1, a member of the nuclear factor of activated $\mathrm{T}$ cells family which functions as a key suppressor of OA, but we did not find evidence for differential expression in our samples $(2.13 \pm 0.4$ relative units for eroded cartilage versus $1.87 \pm 0.4$ relative units for intact cartilage; $P=0.76$ ) (Figure 2F).

Among structural genes, we found hypermethylation of $2 \mathrm{CpG}$ sites within the body of COL11A2, which encodes the pro- $\alpha 2(\mathrm{XI})$ chain of type XI collagen that maintains the diameter and cohesive strength of cartilage matrices (24). Furthermore, we identified a $75 \%$ reduction in COL11A2 expression in eroded cartilage, which corroborates a functional consequence of this hypermethylation $(3.7 \pm 1$ relative units for eroded cartilage versus $15 \pm 0.8$ relative units for intact cartilage; $P=0.0002$ ) (Figure 2A). FGFR2 is involved in chondrocyte differentiation. We found 12 hypermethy- lated CpGs within $200 \mathrm{bp}$ of the TSS or within the $\mathrm{N}$-Shelf of $\mathrm{CpG}$ islands associated with FGFR2. Concordantly, we found a $77 \%$ reduction in gene expression $(0.43 \pm 0.2$ relative units for eroded cartilage versus $1.8 \pm 0.2$ relative units for intact cartilage; $P=0.01$ ) (Figure 2C).

Differential methylation among many OA susceptibility genes. We next turned our attention to the methylation status of known OA susceptibility genes. We reduced the absolute methylation difference $(\Delta \beta)$ threshold requirement for differential methylation from $15 \%$ to $10 \%$ and found $83 \mathrm{CpG}$ sites among 24 of 64 recently reported susceptibility genes, including ADAM12, ACAN, CALM1, CLIP, COL1A1, COL2A1, COL9A2, COL11A2, DOT1L, ENPP1, ESR1, FRZB, FTO, IGF2, IL10, ILIRN, ITGA6, LRP5, MMP3, NCOR2, SERPINA3, SUPT3H, TGFB1, and TP63 (Table 2).

Gene ontology and network analysis show that differentially methylated genes cluster among known OA effectors. Next, we performed IPA to determine networks, diseases, pathways, biologic functions, and upstream regulators associated with differentially methylated genes. The most highly enriched network included many genes known to be associated with OA, and centered on SMAD3 (Figure 3A). A second network involved several genes associated with $\mathrm{OA}$, including HTRA1, NFATC1, and DLX5 (Figure 3B). The top overrepresented biologic functions were "development of cardiovascular system" $\left(P=3.45 \times 10^{-8} ; \mathrm{n}=44\right.$ genes $)$ and "development of connective tissue" $(P=$ $9.79 \times 10^{-6} ; \mathrm{n}=18$ genes). Interestingly, 5 of the top 7 most highly overrepresented functional categories involved angiogenesis or cardiovascular development. Immunologic functions were overrepresented as well, with "cell movement of T lymphocytes" $\left(P=5.39 \times 10^{-6}\right.$; $\mathrm{n}=14$ genes). The top canonical pathway identified was "regulation of interleukin-2 expression in activated and anergic T lymphocytes" $\left(P=1.05 \times 10^{-3}\right)$ (see Supplementary Table 5, available on the Arthritis \& Rheumatology web site at http://onlinelibrary.wiley.com/doi/ 10.1002/art.38762/abstract), highlighting both the importance of inflammation in OA pathology and the likely contribution of epigenetic control mechanisms to OA inflammation.

Overrepresentation of transforming growth factor $\beta$ (TGF $\beta$ ) and both known and novel miRNA associations among differentially methylated genes, as demonstrated by upstream regulator analysis. Differentially methylated genes were predicted to have a number 
Table 2. CpG sites associated with known OA susceptibility genes*

\begin{tabular}{|c|c|c|c|c|c|c|c|}
\hline $\begin{array}{l}\text { OA susceptibility } \\
\text { gene }\end{array}$ & $\begin{array}{l}\text { Illumina } \mathrm{CpG} \\
\text { ID no. }\end{array}$ & $\Delta \beta$ & $\begin{array}{c}P, \\
\text { FDR corrected }\end{array}$ & $\begin{array}{l}\text { OA susceptibility } \\
\text { gene }\end{array}$ & $\begin{array}{l}\text { Illumina } \mathrm{CpG} \\
\text { ID no. }\end{array}$ & $\Delta \beta$ & $\begin{array}{c}P, \\
\text { FDR corrected }\end{array}$ \\
\hline \multirow[t]{2}{*}{$A D A M 12$} & $\operatorname{cg} 02494582$ & 0.13 & $1.1 \times 10^{-35}$ & DOT1L & cg24430201 & -0.11 & $2.7 \times 10^{-15}$ \\
\hline & $\operatorname{cg} 27158677$ & -0.12 & $1.9 \times 10^{-35}$ & ENPP1 & cg18796704 & 0.12 & $2.1 \times 10^{-14}$ \\
\hline$A G C 1 / A C A N$ & $\operatorname{cg} 03648457$ & 0.10 & $4.8 \times 10^{-11}$ & ESR1 & cg06611115 & 0.12 & $1.1 \times 10^{-35}$ \\
\hline CALM1 & cg18079499 & -0.10 & $2.6 \times 10^{-14}$ & & cg01321962 & -0.10 & $2.4 \times 10^{-10}$ \\
\hline \multirow[t]{2}{*}{ CLIP } & $\operatorname{cg} 16111364$ & 0.10 & $3.4 \times 10^{-13}$ & & cg07189962 & -0.10 & $2.1 \times 10^{-11}$ \\
\hline & cg16434674 & -0.15 & $6.2 \times 10^{-24}$ & $F R Z B$ & cg02931467 & 0.10 & $5.4 \times 10^{-10}$ \\
\hline \multirow[t]{2}{*}{ COL1A1 } & $\operatorname{cg} 14562086$ & -0.11 & $1.8 \times 10^{-12}$ & FTO & cg09243909 & 0.17 & $1.1 \times 10^{-35}$ \\
\hline & $\operatorname{cg} 18618815$ & -0.13 & $7.6 \times 10^{-28}$ & $I G F 2$ & $\operatorname{cg} 02425416$ & -0.13 & $4.7 \times 10^{-22}$ \\
\hline COL $2 A 1$ & $\operatorname{cg} 06118478$ & 0.10 & $1.9 \times 10^{-11}$ & & cg05777976 & -0.11 & $1.9 \times 10^{-12}$ \\
\hline \multirow[t]{3}{*}{ COL9A2 } & $\operatorname{cg} 04100190$ & 0.11 & $2.0 \times 10^{-13}$ & & cg19131227 & -0.10 & $2.2 \times 10^{-13}$ \\
\hline & cg10210510 & 0.12 & $1.1 \times 10^{-35}$ & & cg21532432 & 0.10 & $1.0 \times 10^{-12}$ \\
\hline & cg22799499 & -0.10 & $1.4 \times 10^{-12}$ & IL10 & cg15096505 & 0.14 & $1.1 \times 10^{-35}$ \\
\hline \multirow[t]{30}{*}{ COL11A2 } & $\operatorname{cg} 04249605$ & 0.15 & $1.1 \times 10^{-35}$ & $I L 1 R N$ & cg01467417 & -0.10 & $1.0 \times 10^{-10}$ \\
\hline & cg08733307 & 0.14 & $1.1 \times 10^{-35}$ & ITGA6 & cg22061832 & -0.14 & $8.5 \times 10^{-20}$ \\
\hline & cg14683730 & 0.13 & $1.1 \times 10^{-35}$ & $L R P 5$ & cg08356705 & -0.19 & $1.9 \times 10^{-35}$ \\
\hline & $\operatorname{cg} 03260211$ & 0.15 & $1.1 \times 10^{-35}$ & & cg07985116 & 0.12 & $1.1 \times 10^{-35}$ \\
\hline & cg22161893 & 0.19 & $1.1 \times 10^{-35}$ & & cg10771851 & 0.15 & $1.1 \times 10^{-35}$ \\
\hline & cg26998481 & 0.12 & $1.1 \times 10^{-35}$ & & cg13894813 & 0.11 & $3.7 \times 10^{-13}$ \\
\hline & cg12472351 & 0.12 & $1.1 \times 10^{-35}$ & & cg15302350 & 0.11 & $4.0 \times 10^{-12}$ \\
\hline & cg13703714 & 0.14 & $1.1 \times 10^{-35}$ & & cg16409259 & 0.14 & $1.1 \times 10^{-35}$ \\
\hline & $\operatorname{cg} 23704085$ & 0.14 & $1.1 \times 10^{-35}$ & & cg16414472 & 0.14 & $1.1 \times 10^{-35}$ \\
\hline & $\operatorname{cg} 27512176$ & 0.12 & $1.1 \times 10^{-35}$ & & cg20278269 & 0.10 & $1.5 \times 10^{-10}$ \\
\hline & cg17560929 & 0.11 & $1.1 \times 10^{-35}$ & & cg23949925 & -0.11 & $1.2 \times 10^{-12}$ \\
\hline & cg20592734 & 0.12 & $2.1 \times 10^{-14}$ & & cg13738327 & 0.19 & $1.1 \times 10^{-35}$ \\
\hline & cg12312338 & 0.11 & $4.1 \times 10^{-14}$ & MMP3 & cg16466334 & 0.11 & $2.1 \times 10^{-13}$ \\
\hline & cg22501449 & 0.12 & $5.9 \times 10^{-14}$ & & cg23438592 & 0.10 & $1.1 \times 10^{-13}$ \\
\hline & cg22320183 & 0.11 & $7.7 \times 10^{-14}$ & NCOR2 & cg03406367 & 0.10 & $3.7 \times 10^{-11}$ \\
\hline & $\operatorname{cg} 17636806$ & 0.12 & $1.5 \times 10^{-13}$ & & cg05395187 & 0.10 & $2.6 \times 10^{-12}$ \\
\hline & cg01412022 & 0.11 & $7.1 \times 10^{-13}$ & & cg06242243 & 0.10 & $6.8 \times 10^{-11}$ \\
\hline & $\operatorname{cg} 04137133$ & 0.11 & $1.5 \times 10^{-12}$ & & cg09831875 & 0.10 & $1.1 \times 10^{-35}$ \\
\hline & $\operatorname{cg} 00669594$ & 0.11 & $3.3 \times 10^{-12}$ & & cg10082088 & 0.12 & $1.1 \times 10^{-35}$ \\
\hline & cg17591573 & 0.11 & $4.2 \times 10^{-12}$ & & cg10849160 & 0.10 & $2.6 \times 10^{-11}$ \\
\hline & cg11411509 & 0.11 & $7.8 \times 10^{-12}$ & & cg11197258 & 0.10 & $3.3 \times 10^{-9}$ \\
\hline & cg22361816 & 0.10 & $3.7 \times 10^{-11}$ & & cg16706240 & 0.10 & $1.6 \times 10^{-9}$ \\
\hline & $\operatorname{cg} 23231727$ & 0.10 & $4.6 \times 10^{-11}$ & & cg20494738 & 0.14 & $1.1 \times 10^{-35}$ \\
\hline & cg01881428 & 0.10 & $8.1 \times 10^{-11}$ & & cg20978380 & -0.10 & $5.6 \times 10^{-10}$ \\
\hline & $\operatorname{cg} 16507569$ & 0.10 & $1.1 \times 10^{-10}$ & & cg 25954028 & -0.10 & $1.5 \times 10^{-12}$ \\
\hline & cg21893764 & 0.10 & $1.7 \times 10^{-10}$ & SERPINA3 & cg06190732 & 0.11 & $1.2 \times 10^{-12}$ \\
\hline & $\operatorname{cg} 00505762$ & 0.10 & $3.8 \times 10^{-10}$ & SUPT3H & cg01946401 & -0.18 & $1.9 \times 10^{-35}$ \\
\hline & cg24311693 & 0.10 & $6.1 \times 10^{-10}$ & $T G F B 1$ & cg09926389 & 0.10 & $1.6 \times 10^{-12}$ \\
\hline & cg11835806 & 0.10 & $9.3 \times 10^{-10}$ & TP63 & cg09085792 & 0.10 & $2.5 \times 10^{-10}$ \\
\hline & $\operatorname{cg} 01194674$ & 0.10 & $1.5 \times 10^{-9}$ & & & & \\
\hline
\end{tabular}

\footnotetext{
${ }^{*}$ Requirements for inclusion were $P<0.01$ and absolute methylation difference $(\Delta \beta) \geq 0.10$. OA $=$ osteoarthritis (see Table 1 for other definitions).
}

of miRNA interactions (see Supplementary Table 6, available on the Arthritis \& Rheumatology web site at http://onlinelibrary.wiley.com/doi/10.1002/art.38762/ abstract); the top 3 included miR-128 $\left(P=4.48 \times 10^{-13}\right.$; $\mathrm{n}=50$ genes $)$, the MMP regulator miR-27a $(P=4.15 \times$ $10^{-12} ; \mathrm{n}=43$ genes), and the chondroblast survival regulator miR-9 $\left(P=9.20 \times 10^{-10} ; \mathrm{n}=48\right.$ genes $)$. The most enriched non-miRNAs included the protooncogene $E R G\left(P\right.$ of overlap $=9.49 \times 10^{-6} ; \mathrm{n}=11$ genes $)$, the growth factor and OA effector TGFB1 ( $P$ of overlap $=4.40 \times 10^{-5} ; \mathrm{n}=44$ genes $)$, the cytokine $T N F(P$ of overlap $=1.27 \times 10^{-4} ; \mathrm{n}=41$ genes $)$, the inducer of
MMPs $E L K 1$ ( $P$ of overlap $=4.65 \times 10^{-4} ; \mathrm{n}=5$ genes $)$, and the TGF $\beta$-induced signaling molecule SMAD2 ( $P$ of overlap $=5.48 \times 10^{-4} ; \mathrm{n}=6$ genes $)$.

High correlation of several CpG sites with disease activity as measured by histopathologic score. Finally, to study whether $\mathrm{CpG}$ methylation may be correlated with OA progression, we performed histologic modified Mankin scoring on a subset of our samples $(20,25)$. These had a variety of grades of OA; all eroded portions had significantly higher scores than intact portions within the same joint, confirming our gross categorizations (see Supplementary Table 2, avail- 

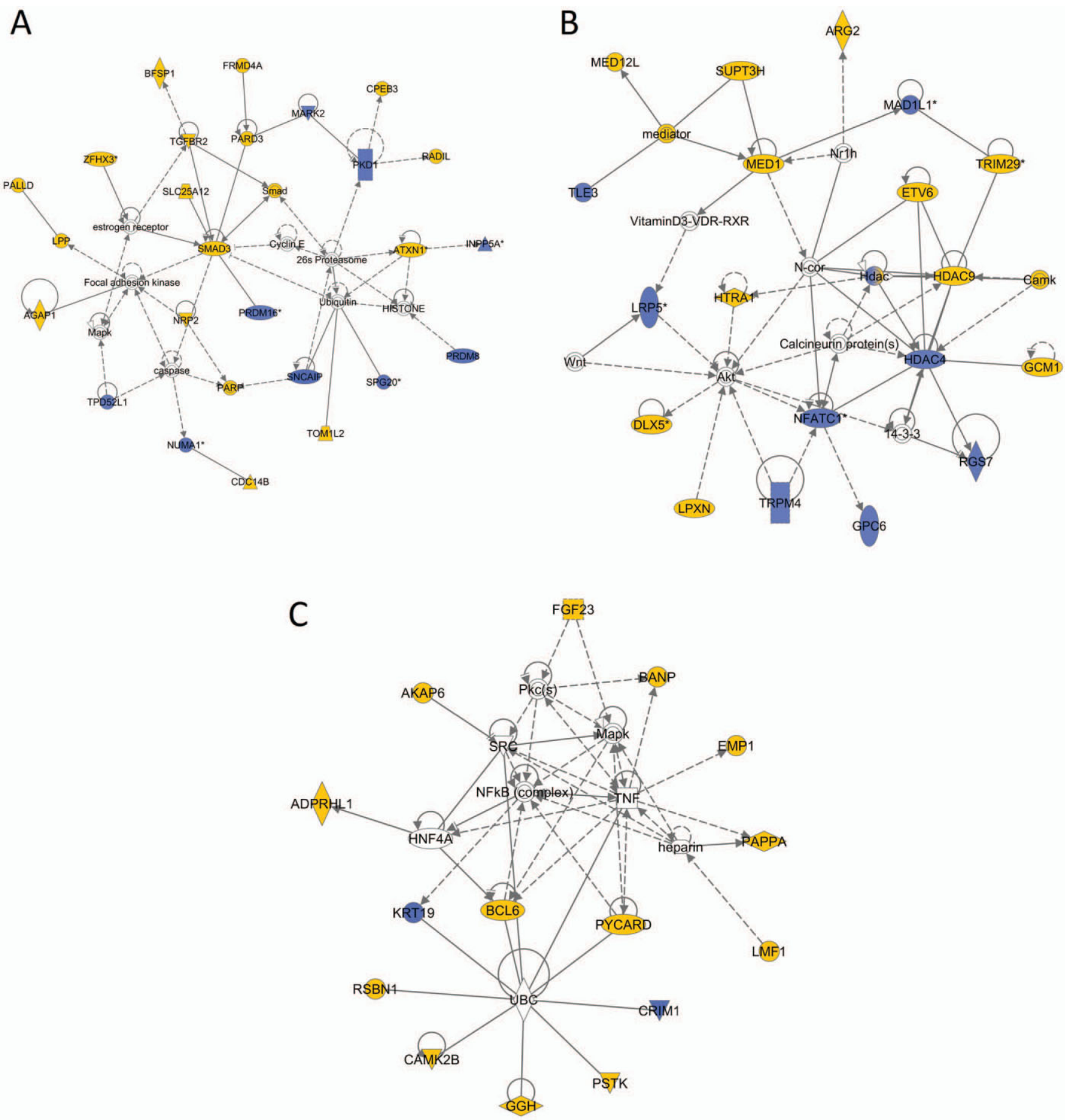

Figure 3. Pathways containing differentially methylated genes. A, Pathway centered upon SMAD3. B, Pathway centered upon Akt. Genes in yellow are hypomethylated. Genes in blue are hypermethylated. C, Pathway enriched in genes with differentially methylated CpG sites highly correlated with the histopathologic Mankin score. Genes in blue are those whose methylation correlated positively with the Mankin score. Genes in yellow are those whose methylation correlated negatively with the Mankin score. Color figure can be viewed in the online issue, which is available at http://onlinelibrary.wiley.com/doi/10.1002/art.38762/abstract.

able on the Arthritis \& Rheumatology web site at http:// onlinelibrary.wiley.com/doi/10.1002/art.38762/abstract).
We identified $20 \mathrm{CpG}$ sites that met criteria for significance (Table 3) (correlations for the 4 most highly 
Table 3. CpG sites most highly correlated with histopathologic modified Mankin score*

\begin{tabular}{|c|c|c|c|c|c|c|}
\hline $\mathrm{R}^{2}$ & $\Delta \beta$ & $\begin{array}{l}\text { Illumina } \mathrm{CpG} \\
\text { ID no. }\end{array}$ & Correlation & Gene symbol & $\begin{array}{l}\text { UCSC location } \\
\text { group }\end{array}$ & $\begin{array}{l}\text { UCSC island } \\
\text { group }\end{array}$ \\
\hline 0.91 & 0.36 & $\operatorname{cg} 14830791$ & - & - & - & - \\
\hline 0.90 & 0.34 & $\operatorname{cg} 06164260$ & - & $B C L 6$ & $5^{\prime}$-UTR, TSS 200 & N-Shore \\
\hline 0.89 & 0.15 & cg01918114 & - & $L M F 1$ & Gene body & - \\
\hline 0.88 & 0.45 & $\operatorname{cg} 11869862$ & + & - & - & N-Shelf \\
\hline 0.88 & 0.29 & cg24601522 & - & - & - & - \\
\hline 0.88 & 0.26 & cg02331262 & - & $B A N P$ & $5^{\prime}$-UTR, $5^{\prime}$-UTR & N-Shelf \\
\hline 0.87 & 0.30 & $\operatorname{cg} 01776691$ & - & $C A M K 2 B$ & Gene body & S-Shore \\
\hline 0.87 & 0.42 & $\operatorname{cg} 08428188$ & - & - & - & Island \\
\hline 0.87 & 0.33 & $\operatorname{cg} 00242786$ & - & ADPRHL1 & TSS 1,500 & - \\
\hline 0.86 & 0.44 & $\operatorname{cg} 08189448$ & - & PAPPA & TSS 200 & $\mathrm{~N}$-Shore \\
\hline 0.86 & 0.25 & $\operatorname{cg} 10764440$ & - & $F G F 23$ & TSS 1,500 & - \\
\hline 0.86 & 0.20 & $\operatorname{cg} 12491114$ & + & KRT19 & TSS 1,500 & S-Shore \\
\hline 0.86 & 0.17 & cg05507566 & + & CRIM1 & Gene body & - \\
\hline 0.85 & 0.39 & cg18849583 & - & AKAP6 & $5^{\prime}$-UTR & - \\
\hline 0.85 & 0.44 & cg19009132 & - & $E M P 1$ & $5^{\prime}$-UTR & - \\
\hline 0.85 & 0.55 & cg24075412 & - & $H R N B P 3$ & TSS 1,500 & - \\
\hline 0.85 & 0.22 & $\operatorname{cg} 00642679$ & - & RSBN1 & TSS 1,500 & S-Shore \\
\hline 0.85 & 0.23 & $\operatorname{cg} 04220482$ & - & $G G H$ & TSS 1,500 & S-Shore \\
\hline 0.85 & 0.17 & $\operatorname{cg} 07461837$ & - & PYCARD & TSS 1,500 & S-Shore \\
\hline 0.85 & 0.29 & cg09419900 & - & PSTK & TSS 200 & N-Shore \\
\hline
\end{tabular}

* $\mathrm{R}^{2}=$ linear coefficient of correlation (see Table 1 for other definitions).

correlated of these $\mathrm{CpG}$ sites are shown in Supplementary Figure 3, available on the Arthritis \& Rheumatology web site at http://onlinelibrary.wiley.com/doi/ 10.1002/art.38762/abstract). Among these, 17 were negatively correlated and 3 were positively correlated with histologic grade. IPA revealed a single network linking 16 of the 17 associated genes, with hubs including OA-associated $N F \kappa B, M a p K, T N F$, and $P k c$ (Figure 3C).

\section{DISCUSSION}

We performed the first genome-wide DNA methylation analysis of matched eroded and intact cartilage tissue from hips of 24 OA patients, offering a unique insight into the progression of this disease. This experimental design reflects a significant departure from previous methylation studies, which have used control tissue from disease-free cadaveric donors or patients undergoing surgical procedures correcting femur neck fractures, a population enriched in osteoporosis, a practice that may introduce its own set of confounding epigenetic aberrations. Our approach lessens the impact of unknown confounders and reduces the contribution of unknown genetic variation, but it does have inherent limitations. In particular, our study design is unable to detect methylation changes from early disease or differential methylation present throughout the affected joint, as most of our intact cartilage samples had at least minimal damage as evidenced by modified Mankin scores $>0$, which may explain the lack of concordance of our findings with a previously reported knee OA genomewide methylation study that used disease-free controls.

Our experiments revealed several significant findings. First, we found evidence for differential methylation among several genes that are intimately involved in OA and among many with associated differential expression, pointing toward a contribution of epigenetic dysregulation to their pathogenic effects. Second, we found moderate differential methylation among more than one-third of known OA susceptibility genes, suggesting DNA methylation as an alternate method for disruption of their activity. Third, network analysis identified pathways enriched in differentially methylated genes with hubs of known OA effectors, and upstream regulator analysis was highly enriched in OAlinked TGFB1 and ERG as well as in both known and novel miRNAs. Finally, we described several $\mathrm{CpG}$ sites whose methylation levels were highly correlated with the histopathologic disease severity score, highlighting the continuous nature of at least some epigenetic marks during disease progression.

We identified $550 \mathrm{CpGs}$ that met our criteria for differential methylation (Table 1 and Figure 1) (see Supplementary Table 7, available on the Arthritis \& Rheumatology web site at http://onlinelibrary.wiley.com/ doi/10.1002/art.38762/abstract), associated with 312 unique genes, enriched in enhancers, previously hypothesized as alternatives to promoters for epigenetic regulatory control (26). Only 3 of these genes were identified 
in a previous knee OA genome-wide methylation study (RUNX1, MGAT1, and WIPF1) (19).

We observed novel differential methylation of several genes involved in OA pathogenesis. Hypomethylated FURIN processes a variety of ADAMTS molecules involved in OA collagen degradation (27). Indeed, inhibition of furin and other like enzymes is sufficient to significantly reduce collagen breakdown and the levels of active collagenase and MMP-2 in vitro (28). Furin is also required to process TGF $\beta 1$ into its active form (29), and inhibition of TGF $\beta 1$ in subchondral bone attenuates OA (30).

The relationship between $R U N X 1$ and $\mathrm{OA}$ is unclear. A previous knee OA genome-wide DNA methylation study identified $R U N X 1$ as the most hypomethylated CpG site in its assay (19). Despite its epigenetic status, several studies have demonstrated, counterintuitively, that RUNX-1 is underexpressed in OA chondrocytes $(31,32)$, which we confirmed in our RT-PCR analysis. Further study to characterize other epigenetic modifications of the local chromatin environment (e.g., histone methylation/acetylation) is needed to explore these seemingly contradictory findings. Our analysis also identified hypomethylation of $R U N X 2$, which is known to be overexpressed in OA chondrocytes. RUNX-2 cooperates with CCAAT/enhancer binding protein $\beta$ to drive MMP-13, a major contributor to cartilage degradation $(33,34)$. Further, it is directly downstream of the MEK/ERK pathway $(35,36)$ and immediately upstream of the MMP ADAMTS-5, both of which have wellestablished roles in OA (37). This hypomethylation corroborates previously observed overexpression of RUNX-2 in OA chondrocytes (34), although we did not find this among our samples.

We also identified hypomethylation of $D L X 5$, which induces the expression of RUNX-2 in animal models (23). We found both hypomethylation and overexpression of HTRA1, which is elevated in synovial fluid and cartilage explants from OA patients $(38,39)$ where it functions to degrade multiple components of the articular cartilage matrix $(38,40)$, releasing MMPs. These findings strongly suggest an epigenetic role in its pathologic expression. NFATC1, a key suppressor of OA, was hypermethylated. This is consistent with previous studies showing reduced NFATC1 expression in OA cartilage (41), although we did not find a significant change in expression among our samples.

Among structural genes, we found evidence for hypermethylation and reduced expression of the type XI collagen gene COL11A2. Mutations within COL11A2 have been associated with a number of hereditary osteochondrodysplasias, featuring severe, early-onset OA de- generative joint disease (42). Interestingly, polymorphisms within COL11A2 have been associated with spinal degenerative disease, but not with OA of the hip or knee (43). Our results suggest epigenetically mediated silencing with effects similar to those caused by genetic mutation. Hypermethylated FGFR2 is also implicated in several genetic craniosynostosis syndromes and is significantly up-regulated during chondrocyte differentiation (44). In addition, we found significant reduction in FGFR2 expression. These both suggest examples of epigenetically mediated gene alterations mimicking the phenotype of genetic mutations.

Upon initial review, among 64 genetic susceptibility loci validated in 3 recent meta-analyses (7-9), only 3 associated genes (CLIP, COL11A2, and SUPT3H) met criteria for differential methylation. This was likely due to our strict $15 \%$ absolute methylation difference requirement, as relaxing this to $10 \%$ revealed a remarkable 83 differentially methylated $\mathrm{CpG}$ sites among 24 known OA susceptibility genes. This is striking; by this definition, $38 \%$ of the known OA susceptibility genes are associated with at least moderate differential DNA methylation (Table 2). Much like findings for COL11A2 and $F G F R 2$, this suggests that gene disruption leading to disease phenotype may be accomplished either by genetic mutation or epigenetic dysregulation.

IPA revealed that many differentially methylated genes are present in networks known to be involved in OA. The first network identified centered on SMAD3 (itself hypomethylated in our assay), which functions in a highly correlated manner with TGF $\beta$ to maintain articular cartilage (45) (Figure 3A). A second network included the aforementioned NFATC1, HTRA1, and $D L X 5$. Akt was central in this network, which upregulates MMP production through hypoxia-inducible factor $2 \alpha$ and NF- $\kappa \mathrm{B}$ and contributes to OA pathology (Figure 3B) (46). Both of these networks demonstrate likely indirect effects of differentially methylated genes on known OA effector pathways.

Several IPAs identified upstream regulators as enriched among differentially methylated genes (see Supplementary Table 5, available on the Arthritis \& Rheumatology web site at http://onlinelibrary.wiley.com/ doi/10.1002/art.38762/abstract). Of the 5 top miRNAs, 2 have previously reported associations with OA. These are miR-9, overexpressed 8-fold in OA cartilage (47), and miR-27a, linked to MMP-13 and insulin-like growth factor binding protein 5 in vitro (48). The other miRNAs we found enriched among differentially methylated genes are novel associations, and these deserve further study. The most highly enriched non-miRNA upstream regulator we identified was the Ets-related gene $E R G$, 
which plays a crucial role in cartilage development through chondrocyte growth and hypertrophy regulation (49). Also highly enriched was TGFB1, present in $11 \%$ $(n=44)$ of differentially methylated genes mapped, with a $P$ value of $4.4 \times 10^{-5}$. Zhen et al recently described chondroprotection in an anterior cruciate ligament destabilization mouse model of OA via both TGF $\beta$ receptor knockout and anti-TGF $\beta$ antibody treatment of subchondral bone, which was linked to a reduction in mesenchymal stem cell recruitment to the site of injury (30). These observed effects might be influenced by epigenetic dysregulation of TGF $\beta$-targeted genes.

Finally, we identified several $\mathrm{CpG}$ sites whose methylation status was highly correlated with the histologic grade of OA (Table 3) (see Supplementary Figure 3, available on the Arthritis \& Rheumatology web site at http://onlinelibrary.wiley.com/doi/10.1002/art.38762/ abstract). All of these genes, with the exception of $F G F 23$, represent novel associations with OA. Interestingly, none met our criteria for significant differential methylation in the larger categorical experiment. IPA identified a single associated network that contained 15 of the 17 differentially methylated genes; the key hubs of this network are known OA- and inflammationassociated proteins and transcription factors including Mapk, TNF, and Pkc (Figure 3C). This finding of a strong association of methylation with histologic grade gives hope for the future identification of biomarkers of disease activity and provides evidence that epigenetic contributions to OA pathology may exist along a continuum, rather than being a binary phenomenon.

In summary, our present set of experiments constituted the first genome-wide DNA methylation study in hip OA using a unique method comparing eroded and intact cartilage from the same joint. We demonstrated a number of differentially methylated $\mathrm{CpG}$ sites, several of these associated with OA-implicated genes, and we offered the first description of differential methylation among OA susceptibility genes. Furthermore, we identified a number of functional networks highly enriched in differentially methylated genes with well-known OA regulators as hubs. Finally, we offered a novel description of strong correlation between the methylation level of several $\mathrm{CpG}$ sites and the histologic grade of OA cartilage, and we found that these associated genes cluster in a single network containing well-known OA hubs. These findings offer further evidence that $\mathrm{OA}$ is a complex disease involving complex genomic-epigenomic interactions.

\section{ACKNOWLEDGMENTS}

We gratefully acknowledge Bradley J. Margo, MD and James D. Mitchell, MD (McBride Orthopedic Hospital, Okla- homa City) for their efforts in patient recruitment and sample collection for this study. We additionally acknowledge Timothy Griffin, PhD (Oklahoma Medical Research Foundation) for critically reviewing the manuscript before submission.

\section{AUTHOR CONTRIBUTIONS}

All authors were involved in drafting the article or revising it critically for important intellectual content, and all authors approved the final version to be published. Drs. Jeffries and Sawalha had full access to all of the data in the study and take responsibility for the integrity of the data and the accuracy of the data analysis.

Study conception and design. Jeffries, James, Sawalha.

Acquisition of data. Jeffries, Donica, Baker, Humphrey.

Analysis and interpretation of data. Jeffries, Stevenson, Annan, James, Sawalha.

\section{REFERENCES}

1. Dieppe PA, Lohmander LS. Pathogenesis and management of pain in osteoarthritis. Lancet 2005;365:965-73.

2. Guccione AA, Felson DT, Anderson JJ, Anthony JM, Zhang Y, Wilson PW, et al. The effects of specific medical conditions on the functional limitations of elders in the Framingham Study. Am J Public Health 1994;84:351-8.

3. Yelin E, Murphy L, Cisternas MG, Foreman AJ, Pasta DJ, Helmick CG. Medical care expenditures and earnings losses among persons with arthritis and other rheumatic conditions in 2003, and comparisons with 1997. Arthritis Rheum 2007;56: 1397-407.

4. Losina E, Thornhill TS, Rome BN, Wright J, Katz JN. The dramatic increase in total knee replacement utilization rates in the United States cannot be fully explained by growth in population size and the obesity epidemic. J Bone Joint Surg Am 2012;94: 201-7.

5. Sacks JJ, Luo YH, Helmick CG. Prevalence of specific types of arthritis and other rheumatic conditions in the ambulatory health care system in the United States, 2001-2005. Arthritis Care Res (Hoboken) 2010;62:460-4.

6. Sandell LJ. Etiology of osteoarthritis: genetics and synovial joint development. Nat Rev Rheumatol 2012;8:77-89.

7. arcOGEN Consortium and arcOGEN Collaborators. Identification of new susceptibility loci for osteoarthritis ( $\operatorname{arcOGEN})$ : a genome-wide association study. Lancet 2012;380:815-23.

8. Rodriguez-Fontenla C, Calaza M, Evangelou E, Valdes AM, Arden N, Blanco FJ, et al. Assessment of osteoarthritis candidate genes in a meta-analysis of 9 genome-wide association studies. Arthritis Rheumatol 2014;66:940-9.

9. Evangelou E, Kerkhof HJ, Styrkarsdottir U, Ntzani EE, Bos SD, Esko T, et al.A meta-analysis of genome-wide association studies identifies novel variants associated with osteoarthritis of the hip. Ann Rheum Dis 2013. E-pub ahead of print.

10. Jeffries MA, Dozmorov M, Tang Y, Merrill JT, Wren JD, Sawalha AH. Genome-wide DNA methylation patterns in $\mathrm{CD}^{+}{ }^{+} \mathrm{T}$ cells from patients with systemic lupus erythematosus. Epigenetics 2011;6:593-601.

11. Jeffries MA, Sawalha AH. Epigenetics in systemic lupus erythematosus: leading the way for specific therapeutic agents. Int J Clin Rheumatol 2011;6:423-39.

12. Coit P, Jeffries M, Altorok N, Dozmorov MG, Koelsch KA, Wren $\mathrm{JD}$, et al. Genome-wide DNA methylation study suggests epigenetic accessibility and transcriptional poising of interferonregulated genes in naive $\mathrm{CD} 4+\mathrm{T}$ cells from lupus patients. J Autoimmun 2013;43:78-84.

13. Liu Y, Aryee MJ, Padyukov L, Fallin MD, Hesselberg E, Runarsson A, et al. Epigenome-wide association data implicate DNA methylation as an intermediary of genetic risk in rheumatoid arthritis. Nat Biotechnol 2013;31:142-7. 
14. de la Rica L, Urquiza JM, Gomez-Cabrero D, Islam AB, LopezBigas $\mathrm{N}$, Tegner $\mathrm{J}$, et al. Identification of novel markers in rheumatoid arthritis through integrated analysis of DNA methylation and microRNA expression. J Autoimmun 2013;41:6-16.

15. Bottini N, Firestein GS. Epigenetics in rheumatoid arthritis: a primer for rheumatologists. Curr Rheumatol Rep 2013;15:372.

16. Roach HI, Yamada N, Cheung KS, Tilley S, Clarke NM, Oreffo $\mathrm{RO}$, et al. Association between the abnormal expression of matrix-degrading enzymes by human osteoarthritic chondrocytes and demethylation of specific $\mathrm{CpG}$ sites in the promoter regions. Arthritis Rheum 2005;52:3110-24.

17. Cheung KS, Hashimoto K, Yamada N, Roach HI. Expression of ADAMTS-4 by chondrocytes in the surface zone of human osteoarthritic cartilage is regulated by epigenetic DNA demethylation. Rheumatol Int 2009;29:525-34.

18. Iliopoulos D, Malizos KN, Tsezou A. Epigenetic regulation of leptin affects MMP-13 expression in osteoarthritic chondrocytes: possible molecular target for osteoarthritis therapeutic intervention. Ann Rheum Dis 2007;66:1616-21.

19. Fernandez-Tajes J, Soto-Hermida A, Vazquez-Mosquera ME, Cortes-Pereira E, Mosquera A, Fernandez-Moreno M, et al. Genome-wide DNA methylation analysis of articular chondrocytes reveals a cluster of osteoarthritic patients. Ann Rheum Dis 2014;73:668-77.

20. Mankin HJ, Dorfman H, Lippiello L, Zarins A. Biochemical and metabolic abnormalities in articular cartilage from osteo-arthritic human hips. II. Correlation of morphology with biochemical and metabolic data. J Bone Joint Surg Am 1971;53:523-37.

21. Lewin J, Schmitt AO, Adorjan P, Hildmann T, Piepenbrock C Quantitative DNA methylation analysis based on four-dye trace data from direct sequencing of PCR amplificates. Bioinformatics 2004;20:3005-12.

22. Moldovan F, Pelletier JP, Mineau F, Dupuis M, Cloutier JM, Martel-Pelletier J. Modulation of collagenase 3 in human osteoarthritic cartilage by activation of extracellular transforming growth factor $\beta$ : role of furin convertase. Arthritis Rheum 2000; 43:2100-9.

23. Lee MH, Kim YJ, Yoon WJ, Kim JI, Kim BG, Hwang YS, et al. Dlx5 specifically regulates Runx2 type II expression by binding to homeodomain-response elements in the Runx2 distal promoter. J Biol Chem 2005;280:35579-87.

24. Li Y, Lacerda DA, Warman ML, Beier DR, Yoshioka H, Ninomiya Y, et al. A fibrillar collagen gene, Col11a1, is essential for skeletal morphogenesis. Cell 1995;80:423-30.

25. Pearson RG, Kurien T, Shu KS, Scammell BE. Histopathology grading systems for characterisation of human knee osteoarthritis -reproducibility, variability, reliability, correlation, and validity. Osteoarthritis Cartilage 2011;19:324-31.

26. De Andres MC, Imagawa $\mathrm{K}$, Hashimoto K, Gonzalez A, Roach $\mathrm{HI}$, Goldring MB, et al. Loss of methylation in $\mathrm{CpG}$ sites in the $N F-\kappa B$ enhancer elements of inducible nitric oxide synthase is responsible for gene induction in human articular chondrocytes. Arthritis Rheum 2013;65:732-42.

27. Longpre JM, McCulloch DR, Koo BH, Alexander JP, Apte SS, Leduc R. Characterization of proADAMTS5 processing by proprotein convertases. Int J Biochem Cell Biol 2009;41:1116-26.

28. Milner JM, Rowan AD, Elliott SF, Cawston TE. Inhibition of furin-like enzymes blocks interleukin-1 $\alpha /$ oncostatin M-stimulated cartilage degradation. Arthritis Rheum 2003;48:1057-66.

29. Dubois CM, Blanchette F, Laprise MH, Leduc R, Grondin F, Seidah NG. Evidence that furin is an authentic transforming growth factor- $\beta 1$-converting enzyme. Am J Pathol 2001;158: $305-16$.

30. Zhen G, Wen C, Jia X, Li Y, Crane JL, Mears SC, et al. Inhibition of TGF- $\beta$ signaling in mesenchymal stem cells of subchondral bone attenuates osteoarthritis. Nat Med 2013;19:704-12.

31. Karlsson C, Dehne T, Lindahl A, Brittberg M, Pruss A, Sittinger $\mathrm{M}$, et al. Genome-wide expression profiling reveals new candidate genes associated with osteoarthritis. Osteoarthritis Cartilage 2010; 18:581-92.
32. Yano F, Hojo H, Ohba S, Fukai A, Hosaka Y, Ikeda T, et al. A novel disease-modifying osteoarthritis drug candidate targeting Runx1. Ann Rheum Dis 2013;72:748-53.

33. Hirata M, Kugimiya F, Fukai A, Saito T, Yano F, Ikeda T, et al. $\mathrm{C} / \mathrm{EBP} \beta$ and RUNX2 cooperate to degrade cartilage with MMP-13 as the target and HIF- $2 \alpha$ as the inducer in chondrocytes. Hum Mol Genet 2012;21:1111-23.

34. Wang X, Manner PA, Horner A, Shum L, Tuan RS, Nuckolls GH Regulation of MMP-13 expression by RUNX2 and FGF2 in osteoarthritic cartilage. Osteoarthritis Cartilage 2004;12:963-73.

35. Hsieh YS, Yang SF, Lue KH, Chu SC, Li TJ, Lu KH. Upregulation of urokinase-type plasminogen activator and inhibitor and gelatinase expression via 3 mitogen-activated protein kinases and PI3K pathways during the early development of osteoarthritis. J Rheumatol 2007;34:785-93.

36. Prasadam I, Mao X, Shi W, Crawford R, Xiao Y. Combination of MEK-ERK inhibitor and hyaluronic acid has a synergistic effect on anti-hypertrophic and pro-chondrogenic activities in osteoarthritis treatment. J Mol Med 2013;91:369-80.

37. Lin AC, Seeto BL, Bartoszko JM, Khoury MA, Whetstone H, Ho $\mathrm{L}$, et al. Modulating hedgehog signaling can attenuate the severity of osteoarthritis. Nat Med 2009;15:1421-5.

38. Grau S, Richards PJ, Kerr B, Hughes C, Caterson B, Williams AS, et al. The role of human HtrA1 in arthritic disease. J Biol Chem 2006;281:6124-9.

39. Hu SI, Carozza M, Klein M, Nantermet P, Luk D, Crowl RM. Human HtrA, an evolutionarily conserved serine protease identified as a differentially expressed gene product in osteoarthritic cartilage. J Biol Chem 1998;273:34406-12.

40. Chamberland A, Wang E, Jones AR, Collins-Racie LA, LaVallie ER, Huang Y, et al. Identification of a novel HtrA1-susceptible cleavage site in human aggrecan: evidence for the involvement of HtrA1 in aggrecan proteolysis in vivo. J Biol Chem 2009;284: 27352-9.

41. Greenblatt MB, Ritter SY, Wright J, Tsang K, Hu D, Glimcher LH, et al. NFATc1 and NFATc2 repress spontaneous osteoarthritis. Proc Natl Acad Sci U S A 2013;110:19914-9.

42. Vikkula M, Mariman EC, Lui VC, Zhidkova NI, Tiller GE, Goldring MB, et al. Autosomal dominant and recessive osteochondrodysplasias associated with the COL11A2 locus. Cell 1995;80: $431-7$.

43. Ryder JJ, Garrison K, Song F, Hooper L, Skinner J, Loke Y, et al. Genetic associations in peripheral joint osteoarthritis and spinal degenerative disease: a systematic review. Ann Rheum Dis 2008; 67;584-91.

44. Sekiya I, Vuoristo JT, Larson BL, Prockop DJ. In vitro cartilage formation by human adult stem cells from bone marrow stroma defines the sequence of cellular and molecular events during chondrogenesis. Proc Natl Acad Sci U S A 2002;99: 4397-402.

45. Yang X, Chen L, Xu X, Li C, Huang C, Deng CX. TGF- $\beta /$ Smad3 signals repress chondrocyte hypertrophic differentiation and are required for maintaining articular cartilage. J Cell Biol 2001;153: $35-46$.

46. Lin TH, Tang CH, Wu K, Fong YC, Yang RS, Fu WM. 15-deoxy$\Delta^{12,14}$-prostaglandin-J2 and ciglitazone inhibit TNF- $\alpha$-induced matrix metalloproteinase 13 production via the antagonism of NF- $\kappa \mathrm{B}$ activation in human synovial fibroblasts. J Cell Physiol 2011;226: 3242-50.

47. Jones SW, Watkins G, Le Good N, Roberts S, Murphy CL, Brockbank SM, et al. The identification of differentially expressed microRNA in osteoarthritic tissue that modulate the production of TNF- $\alpha$ and MMP13. Osteoarthritis Cartilage 2009;17:464-72.

48. Tardif G, Hum D, Pelletier JP, Duval N, Martel-Pelletier J. Regulation of the IGFBP-5 and MMP-13 genes by the microRNAs miR-140 and miR-27a in human osteoarthritic chondrocytes. BMC Musculoskelet Disord 2009;10:148.

49. Iwamoto M, Higuchi Y, Enomoto-Iwamoto M, Kurisu K, Koyama $\mathrm{E}$, Yeh $\mathrm{H}$, et al. The role of ERG (ets related gene) in cartilage development. Osteoarthritis Cartilage 2001;9 Suppl A:S41-7. 